•研究报告・

\title{
格氏栲天然林林窗植物物种多样性与系统发育多 样性
}

陈博 $1,2,3$, 江蓝 $1,2,3$, 谢子扬 ${ }^{1,2,3}$, 李阳娣 ${ }^{1}$, 李佳萱 ${ }^{1}$, 李梦佳 ${ }^{1,2,3}$, 魏晨思 ${ }^{1,2,3}$, 邢 聪 $1,2,3$, 刘 金福 $1,2,3$, 何中声(1) 1,2,3*

1. 福建农林大学林学院, 福州 350002；2. 福建农林大学海峡自然保护区研究中心，福州 350002; 3. 生态与资源统计福建省高校重点实验 室, 福州 350002

摘要: 林窗环境异质性导致群落物种多样性与系统发育多样性(phylogenetic diversity, PD)存在差异, 研究不同大小的林窗中 群落的物种多样性与系统发育多样性有助于揭示林下生物多样性的形成及维持机制。本文以格氏栲(Castanopsis kawakamii) 天然林为研究对象, 通过Pearson相关性分析与广义线性模型探讨了林窗内物种多样性与系统发育多样性间的相互关系及其 环境影响因素。结果表明: (1)大林窗(面积 $\left.>200 \mathrm{~m}^{2}\right)$ 植物种类及多度均高于中林窗 $\left(50 \mathrm{~m}^{2} \leq\right.$ 面积 $<100 \mathrm{~m}^{2}$ )、小林窗 $\left(30 \mathrm{~m}^{2} \leq\right.$ 面积 $<50 \mathrm{~m}^{2}$ ) 和非林窗 (面积 $=100 \mathrm{~m}^{2}$ )。大林窗群落系统发育结构趋于发散, 中、小林窗和非林窗群落系统发育结构受到生 境过滤和竞争排斥综合作用。(2)群落系统发育多样性指数与物种丰富度(species richness, SR)、Margalef丰富度指数和 Shannon-Wiener指数均呈显著正相关, 这与林窗内稀有种种类组成多于优势种有关。(3)林窗面积对物种多样性存在显著正效 应; 土壤全氮含量对系统发育多样性和系统发育结构存在显著正效应。林窗形成提高了格氏栲天然林群落物种多样性和系统 发育多样性，林窗面积与土壤全氮共同驱动了格氏栲天然林林窗物种多样性和系统发育多样性的变化。

关键词: 格氏栲; 林窗; 物种多样性; 系统发育多样性; 环境因素

陈博，江蓝，谢子扬，李阳娣，李佳萱，李梦佳，魏晨思，邢聪，刘金福，何中声 (2021) 格氏栲天然林林窗植物物种多样性与系统发育多样性。 生物多样性, 29, 439-448. doi: 10.17520/biods.2020399.

Chen B, Jiang L, Xie ZY, Li YD, Li JX, Li MJ, Wei CS, Xing C, Liu JF, He ZS (2021) Taxonomic and phylogenetic diversity of plants in a Castanopsis kawakamii natural forest. Biodiversity Science, 29, 439-448. doi: 10.17520/biods.2020399.

\section{Taxonomic and phylogenetic diversity of plants in a Castanopsis kawakamii natural forest}

Bo Chen ${ }^{1,2,3}$, Lan Jiang ${ }^{1,2,3}$, Ziyang Xie ${ }^{1,2,3}$, Yangdi $\mathrm{Li}^{1}$, Jiaxuan $\mathrm{Li}^{1}$, Mengjia $\mathrm{Li}^{1,2,3}$, Chensi Wei ${ }^{1,2,3}$, Cong Xing ${ }^{1,2,3}$, Jinfu $\mathrm{Liu}^{1,2,3}$, Zhongsheng $\mathrm{He}^{\left(11,2,3^{*}\right.}$

1 College of Forestry, Fujian Agriculture and Forestry University, Fuzhou 350002

2 Cross-Strait Nature Reserve Research Center, Fujian Agriculture and Forestry University, Fuzhou 350002

3 Key Laboratory of Fujian Universities for Ecology and Resource Statistics, Fuzhou 350002

\section{ABSTRACT}

Aims: Environmental heterogeneity of forest gaps leads to variation in taxonomic and phylogenetic diversity of trees in these areas. Studying tree diversity in different sizes of forest gap communities can help to reveal the mechanisms that drive the formation and maintenance of biodiversity. This study took Castanopsis kawakamii gaps as the research object, and aimed to reveal the relationship between the taxonomic and phylogenetic diversity of plants and its environmental influence factors.

Methods: We examined different sizes of forest gaps in a Castanopsis kawakamii natural forest as to study the taxonomic and phylogenetic diversity of plants, and used a generalized linear model (GLM) to explore the

收稿日期: 2020-10-13; 接受日期: 2021-02-07

基金项目: 国家自然科学基金(31700550; 31770678)、福建省自然科学基金(2019J01367)、福建省林业科技推广项目(2018TG14-2)和福建农林大学科技 创新基金(CXZX2018125)

* 通讯作者 Author for correspondence. E-mail: jxhzs85@126.com 
environmental factors driving the community assembly.

Results: We found that the plant species and plant abundance in large gaps $\left(>200 \mathrm{~m}^{2}\right)$ were higher than those of medium gaps $\left(\left[50 \mathrm{~m}^{2}, 100 \mathrm{~m}^{2}\right)\right)$, small gaps $\left(\left[30 \mathrm{~m}^{2}, 50 \mathrm{~m}^{2}\right)\right)$ and non-gaps $\left(100 \mathrm{~m}^{2}\right)$. The phylogenetic community structure of the large gaps tends to diverge, while that of the medium gaps, small gaps and non-gaps were affected by the combined effect of habitat filtering and competitive exclusion. The phylogenetic community diversity index (PD) was significantly positively correlated with species richness (SR), Margalef index and Shannon-Wiener index, which is related to the higher species composition of sparse species than dense species in forest gaps. Overall, forest gap size had a significantly positive effect on species diversity, and the soil total nitrogen content had a significantly positive effect on community phylogenetic diversity and phylogenetic structure.

Conclusion: The formation of forest gaps increase the taxonomic and phylogenetic diversity of trees in natural forests, with gap size and soil total nitrogen jointly driving tree diversity in these natural forest gaps.

Key words: Castanopsis kawakamii; forest gap; tree taxonomic diversity; phylogenetic diversity; environment factors

林窗(forest gap)是森林群落内林冠层树木个体 死亡而形成的空隙，是森林中常见的小尺度干扰 (Hubbell et al, 1999)。林窗动态与生物多样性密切相 关，通过改变光、温、水和土壤环境因子而影响森 林生态系统循环, 进而促进森林群落演替和物种更 新(Mao et al, 2020)。林窗大小是林窗最基本的特征 之一, 不同大小林窗的环境异质性对群落物种多样 性有重要影响(Lohbeck et al, 2014)。林窗形成可以 促进种子萌发, 增加幼苗更新潜力与幼苗生长, 提 高物种多样性(姚俊宇等, 2017)。物种多样性反映了 一个地区物种丰富度的高低, 对其保护不仅聚焦于 该区域现存的物种丰富度, 区域物种的进化信息也 是重要的保护对象。系统发育多样性从亲缘关系角 度阐释了物种的进化历程。结合系统发育多样性和 物种多样性可为生物多样性保护提供新的视角。

系统发育多样性包含了物种间的系统发育关 系与进化信息, 是生物多样性的重要组成部分, 在 保护生物学研究领域有着重要地位。对南非好望角 植物系统发育多样性和物种丰富度的研究表明, 系 统发育多样性是对物种谱系进化史在特定时空内 生物类群独特性评估的重要指标, 将其运用到生物 多样性保护决策中能为区域群落提供更全面的保 护方案(Forest et al, 2007; Pio et al, 2011)。系统发育 结构是系统发育多样性的补充, 反映了群落构建的 生态学过程, 综合系统发育多样性及系统发育结构 可从进化和生态角度研究物种的组成、起源和演化 过程, 进而促进林下生物多样性的合理保护(慈秀 芹和李捷, 2017)。群落系统发育多样性与系统发育 结构易受环境因素影响(Gastauer et al, 2020), 对长 白山不同海拔梯度群落系统发育多样性与物种多 样性的研究表明, 环境因子是驱动该地区森林群落
系统发育多样性与系统发育结构变化的主要因素 (Huang et al, 2010; Qian et al, 2014)。Myers等(2013) 证明了温带与热带森林的光照与降水能引起群落 构建机制的差异, 在温带森林群落构建过程中环境 过滤占主导地位, 而热带森林中扩散限制占优势。 因此, 从物种多样性、群落系统发育多样性与系统 发育结构及其环境影响因素的角度出发, 更利于特 定区域群落物种的保护。

珍稀濒危植物格氏栲(Castanopsis kawakamii) 是中亚热带南缘特有的壳斗科常绿高大乔木, 仅零 星分布于福建、广东、广西和台湾等地区(何中声等, 2012)。福建三明格氏栲自然保护区有约700 ha以格 氏栲为优势种的天然林分, 有着“世界格氏栲林”之 称(He et al, 2012)。然而受自身生物学特性、种源、 环境和人为干扰等综合作用, 格氏栲种群存在退化 趋势(何中声等, 2012)。目前这片格氏栲种群已过熟, 林下更新困难, 部分林冠层出现严重断层现象, 破 碎化严重, 导致林窗数量增多(何中声等, 2012)。林 窗的形成使得林内微环境存在差异, 潜在改变了群 落物种组成, 进而影响森林植被组成(王进欣和张 一平, 2002)。许多学者先后开展了格氏栲林窗更新 层物种多样性和林窗边缘效应等研究(刘金福等, 2003; 何中声等, 2012; Buajan et al, 2018), 然而对 于格氏栲天然林林窗环境下的物种多样性和系统 发育多样性鲜有报道。为此, 我们以格氏栲天然林 为研究对象, 开展不同大小林窗内物种多样性与系 统发育多样性间关系的研究, 主要探讨: (1)不同大 小林窗物种多样性、系统发育多样性与系统发育结 构之间是否存在差异? (2)物种多样性与系统发育多 样性指数之间相关性如何? (3)哪些环境因子驱动群 落物种多样性、系统发育多样性与系统发育结构的 
形成? 以期综合群落系统发育多样性和物种多样 性特征, 为格氏栲林生物多样性提供科学的保护方 案, 为促进格氏栲林的可持续经营与发展提供理论 依据。

\section{材料与方法}

\section{1 研究区概况}

格氏栲自然保护区位于福建省三明市西南部 $\left(26^{\circ} 10^{\prime}-26^{\circ} 12^{\prime} \mathrm{N}, 117^{\circ} 27^{\prime}-117^{\circ} 30^{\prime} \mathrm{E}\right)$, 海拔范围 180-604 m, 低山丘岭地貌。属于中亚热带季风气候, 年平均气温 $19.2^{\circ} \mathrm{C}$, 年降水量约 $1,600 \mathrm{~mm}$ 。土壤类 型以酸性铁铝土为主, 腐殖层丰富。该地区植物种 类丰富，常见物种有树参(Dendropanax dentiger)、 虎皮楠(Daphniphyllum oldhami)、细枝柃 (Eurya loquaiana)、桂北木姜子(Litsea subcoriacea)、米槠 (Castanopsis carlesii) 、酸味子 (Antidesma japonicum)、狗骨柴(Diplospora dubia)等(He et al, 2012)。

\section{2 样地设置}

以三明格氏栲天然林为研究对象, 根据前期调 查选择代表性林窗样地进行实地调查。利用尼康数 码相机搭配鱼眼镜头在林窗中央垂直向上拍摄照 片, 采用半球面影像法计算林窗面积(胡理乐等, 2010)。依据Hubbell等(1999)对于林窗大小的划分方 法，并根据格氏栲林窗形成原因以及发育期将林窗 划分为大中小 3 个等级。其中, 大林窗 3 个, 面积分 别为 $210.55 \mathrm{~m}^{2}$ (LG1)、200.39 $\mathrm{m}^{2}$ (LG2)和 $207.56 \mathrm{~m}^{2}$ (LG3); 中林窗3个, 面积分别为74.23 $\mathrm{m}^{2}$ (MG1)、 $70.60 \mathrm{~m}^{2}$ (MG2)和74.64 $\mathrm{m}^{2}$ (MG3); 小林窗3个, 面 积分别为 $32.12 \mathrm{~m}^{2}$ (SG1)、31.58 $\mathrm{m}^{2}$ (SG2)和 $36.77 \mathrm{~m}^{2}$ (SG3)。同时, 在郁闭林下设置 3 个 $10 \mathrm{~m} \times 10 \mathrm{~m}$ 的非 林窗(NG)样地作为对照。调查林窗与非林窗样地内 所有胸径大于 $1 \mathrm{~cm}$ 的植株(乔木和灌木), 记录其种 名、胸径、树高等信息。植物种名参考中国在线植 物志(http://frps.iplant.cn/)。

\section{3 环境因子调查与测定}

土壤与空气温度监测采用美国MAXIM iButton

(MAXIM Intergrated, iButton, San Jose, USA) DS1922L-F50型(土壤温度)和DS1923-F5型(空气温 湿度)记录器。在大、中、小以及非林窗样地中央, 空 气温度记录器放置于距离土壤表层垂直高度 $1.5 \mathrm{~m}$ 处, 土壤温度记录器放置于土壤表层以下 $10 \mathrm{~cm}$ 处。
每日从 0 点开始, 每隔 $4 \mathrm{~h}$ 自动记录一次空气与土壤 温度, 以 1 年记录的平均值作为各林窗及非林窗样 地的空气与土壤温度数据。在林窗及非林窗样地的 中央及东南西北四个方位随机选取 3 个采样点, 在 每个采样点分 3 处用土壤环刀(规格 $100 \mathrm{~cm}^{3}$ ) 钻取 0-20 cm 土层土壤, 混合后用于土壤理化性质测定。 土壤理化指标包括土壤含水量、土壤 $\mathrm{pH}$ 值、全碳、 全氮、全磷、全钾、水解氮、有效磷、碳氮比、氮 磷比和碳磷比(表1)。土壤含水量采用环刀法测定; 土壤 $\mathrm{pH}$ 值采用电位法测定; 土壤全碳和全氮采用 碳氮分析仪(VARIO MAX CN Elemental Analyzer, Elementar, German)测定; 土壤全磷和全钾采用电感 耦合等离子体发射光谱仪 (PE OPTIMA 8000, PerkinElmer, USA)测定; 土壤水解氮采用碱解扩散 法测定; 土壤有效磷采用钼锑抗比色法测定; 碳氮 比为土壤全碳与全氮之比; 氮磷比为土壤全氮与全 磷之比; 碳磷比为土壤全碳与全磷之比。详细测定 方法参考《土壤农化分析》(鲍士旦, 2000)。

1.3 物种多样性、系统发育多样性与系统发育结构 物种 $\alpha$ 多样性采用物种丰富度 (SR)、 Shannon-Wiener指数、Margalef丰富度指数、Simpson 优势度指数和Pielou均匀度指数测度。物种进化树 通过R 3.6.1的plantlist包得到科属种列表后，再利用 phylomatic (http://phylodiversity.net/phylomatuc/) 在 线生成植物分类系统APG III以及具有分化时间的 谱系树结构数据, 并整合Zanne进化树骨架, 最后 采用picante包计算系统发育多样性(PD)和系统发育 结构(Faith, 1992; Qian \& Jin, 2014)。系统发育多样 性用于量化群落发育的总枝长。为保证物种个体在 其分布空间的随机性，采用R 3.6.1剔除SR后，再经 随机零模型运算得出标准化PD (SES.PD)值(Mori et al, 2015)。

系统发育结构采用净谱系亲缘关系指数 (net relatedness index, $N R I$ ) 和净最近种间亲缘关系指数 (net nearest taxa index, NTI)测度。NRI指数侧重于物 种之间的相似度, NTI则侧重于相似物种间的影响。 $N R I$ 和NTI计算公式如下:

$$
\begin{aligned}
& N R I=-\frac{\mathrm{MPD}_{\mathrm{s}}-\mathrm{MPD}_{\mathrm{mds}}}{\mathrm{SD}\left(\mathrm{MPD}_{\mathrm{mds}}\right)} \\
& N T I=-\frac{\mathrm{MNTD}_{\mathrm{s}}-\mathrm{MNTD}_{\mathrm{mds}}}{\mathrm{SD}\left(\mathrm{MPD}_{\mathrm{mds}}\right)}
\end{aligned}
$$


表1＼cjkstart格氏栲天然林不同大小林窗和非林窗环境因子指标

Table 1 Index of environmental factors of different forest gaps and non-gaps in Castanopsis kawakamii natual forest

\begin{tabular}{|c|c|c|c|c|}
\hline & 大林窗 Large gap & 中林窗 Middle gap & 小林窗 Small gap & 非林窗 Non-gap \\
\hline 面积 Area $\left(\mathrm{m}^{2}\right)$ & $206.17 \pm 4.26^{\mathrm{a}}$ & $73.16 \pm 1.82^{\mathrm{b}}$ & $33.49 \pm 2.33^{\mathrm{b}}$ & $100 \pm 0.00^{\mathrm{ab}}$ \\
\hline 海拔 Elevation (m) & $216.33 \pm 5.56^{\mathrm{ab}}$ & $199.33 \pm 10.87^{\mathrm{b}}$ & $214.00 \pm 8.98^{\mathrm{ab}}$ & $221.17 \pm 0.17^{\mathrm{a}}$ \\
\hline 坡度 Slope $\left(^{\circ}\right)$ & $27.33 \pm 3.86^{\mathrm{ab}}$ & $29.33 \pm 1.25^{\mathrm{a}}$ & $17.67 \pm 7.41^{\mathrm{bc}}$ & $13.23 \pm 0.09^{c}$ \\
\hline 坡位 Slope position & $2.33 \pm 0.47^{\mathrm{a}}$ & $1.67 \pm 0.94^{\mathrm{a}}$ & $2.00 \pm 0.82^{\mathrm{a}}$ & $1.00 \pm 0.01^{\mathrm{a}}$ \\
\hline 土壤含水量 Soil water content (g/kg) & $302.77 \pm 32.7^{\mathrm{a}}$ & $280.72 \pm 9.39^{\mathrm{a}}$ & $277.90 \pm 11.90^{\mathrm{a}}$ & $269.56 \pm 8.86^{\mathrm{a}}$ \\
\hline 土壤全碳含量 Soil total C content (g/kg) & $17.49 \pm 3.71^{\mathrm{a}}$ & $19.19 \pm 3.69^{\mathrm{a}}$ & $21.49 \pm 5.34^{\mathrm{a}}$ & $23.79 \pm 0.48^{\mathrm{a}}$ \\
\hline 土壤全氮含量 Soil total $\mathrm{N}$ content (g/kg) & $0.99 \pm 0.07^{\mathrm{a}}$ & $1.05 \pm 0.21^{\mathrm{a}}$ & $1.39 \pm 0.5^{\mathrm{a}}$ & $1.48 \pm 0.04^{\mathrm{a}}$ \\
\hline 土壤全磷含量 Soil total P content (g/kg) & $0.62 \pm 0.39^{\mathrm{a}}$ & $0.67 \pm 0.17^{\mathrm{a}}$ & $0.38 \pm 0.08^{\mathrm{a}}$ & $0.18 \pm 0.01^{\mathrm{a}}$ \\
\hline 土壤全钾含量 Soil total K content (mg/L) & $30.9 \pm 0.62^{\mathrm{a}}$ & $22.3 \pm 4.97^{\mathrm{b}}$ & $21.5 \pm 0.67^{\mathrm{b}}$ & $27.17 \pm 0.82^{\mathrm{ab}}$ \\
\hline pH值 $\mathrm{pH}$ value & $3.49 \pm 0.05^{\mathrm{a}}$ & $3.39 \pm 0.04^{\mathrm{a}}$ & $3.52 \pm 0.12^{\mathrm{a}}$ & $3.41 \pm 0.02^{\mathrm{a}}$ \\
\hline 水解氮 Hydrolyzed nitrogen (mg/kg) & $124.45 \pm 8.00^{\mathrm{a}}$ & $128.01 \pm 31.19^{\mathrm{a}}$ & $111.01 \pm 9.02^{\mathrm{a}}$ & $136.41 \pm 3.01^{\mathrm{a}}$ \\
\hline 碳氮比 $\mathrm{C} / \mathrm{N}$ & $17.43 \pm 2.71^{\mathrm{a}}$ & $18.08 \pm 1.08^{\mathrm{a}}$ & $16.66 \pm 1.66^{\mathrm{a}}$ & $16.55 \pm 0.83^{\mathrm{a}}$ \\
\hline 有效磷含量 Available P content (mg/kg) & $5.26 \pm 2.70^{\mathrm{ab}}$ & $11.31 \pm 8.95^{\mathrm{ab}}$ & $19.57 \pm 1.84^{\mathrm{a}}$ & $3.87 \pm 0.20^{\mathrm{b}}$ \\
\hline 年均空气温度 Annual air temperature $\left({ }^{\circ} \mathrm{C}\right)$ & $25.15 \pm 0.53^{\mathrm{a}}$ & $25.20 \pm 0.07^{\mathrm{a}}$ & $24.91 \pm 0.24^{\mathrm{a}}$ & $24.27 \pm 0.48^{\mathrm{a}}$ \\
\hline 年均空气湿度 Annual air humidity (\%) & $90.08 \pm 1.22^{\mathrm{a}}$ & $85.34 \pm 7.14^{\mathrm{a}}$ & $90.14 \pm 2.49^{\mathrm{a}}$ & $91.75 \pm 0.81^{\mathrm{a}}$ \\
\hline 年均土壤温度 Annual soil temperature $\left({ }^{\circ} \mathrm{C}\right)$ & $24.38 \pm 1.11^{\mathrm{a}}$ & $24.25 \pm 0.35^{\mathrm{a}}$ & $23.40 \pm 0.49^{\mathrm{a}}$ & $23.01 \pm 0.47^{\mathrm{a}}$ \\
\hline
\end{tabular}

同行不同字母表示不同大小林窗间环境因子具有显著差异 $(P<0.05)$ 。

Different lowercases in the same row indicate that the environmental factors have significant difference between forest gap sizes $(P<0.05)$.

其中, $\mathrm{SD}$ 指标准差, MPD和MNTD分别表示物种间 的平均成对系统发育距离与平均最近种间系统发 育距离, $\mathrm{MPD}_{\mathrm{s}}$ 和 $\mathrm{MNTD}_{\mathrm{s}}$ 则表示实际观察到的MPD 值和群落内亲缘关系最近的物种之间的平均系统 发育距离, $\mathrm{MPD}_{\mathrm{mds}}$ 和 $M N T D_{\mathrm{mds}}$ 表示通过软件随机模 拟999次的平均谱系距离和最近相邻谱系距离的平 均值。当 $N R I>0, N T I>0$ 时, 系统发育结构聚集, 表 示该群落是趋向于亲缘关系比较近的物种组成; 当 $N R I<0, N T I<0$ 时, 系统发育结构趋于分散, 群落 由亲缘关系较远的物种组成, 竞争排斥是群落构建 的主导因素; 若 $N R I=0, N T I=0$, 代表此群落系统 发育结构随机, 环境与生物因素共同维持物种结构 的多样性(Webb et al, 2002)。

由于林窗面积不一致, 根据物种面积累计曲线 将每个林窗的 SES.PD、Margalef、Simpson、 Shannon-Wiener和Pielou指数及SR根据林窗面积大 小进行标准化处理(即相关指标除以 $\lg$ (面积)进行对 数转换, 面积单位为 $\mathrm{m}^{2}$ ) (孙雀等, 2009; Qian et al, 2019)。由于 NTI和 NRI为标准化指数, 研究中未进行 转换(Qian et al, 2019)。

\section{4 数据分析}

在检验数据符合正态分布后, 采用单因素方差 分析(one-way ANOVA)检验物种多样性与系统发育 多样性指数在不同大小林窗间的差异性。采用
Pearson相关性分析检验物种多样性、系统发育多样 性和系统发育结构间的相互关系。

林窗和非林窗的环境因子经正态分布检验后, 进行Duncan's检验和方差分析。采用Pearson相关性 分析篮选对群落物种多样性、系统发育多样性和系 统发育结构存在显著性的环境因子, 采用广义线性 模型(generalized linear model, GLM)中基于正态分 布的回归模型探讨影响系统发育指数与物种多样 性指数的关键因子。根据AIC (Akaike information criterion)准则笁选 “最优模型” 确定主要驱动因子, 并采用方差膨胀因子(variance inflation factor, VIF) 进行共线性诊断, 剔除VIF > 10的变量后再进行重 新拟合。

数据分析采用R 3.6.1完成, 使用mass包进行单 因素方差分析、正态分布检验，corrplot进行相关分 析, broom包进行广义线性模型分析, picante包进行 零模型分析(R core team, 2019; Mori et al, 2015)。

\section{2 结果}

\section{1 不同大小林窗物种组成}

大林窗内物种丰富度大于15的优势种包括：格 氏栲、木荷(Schima superba)、鼠刺(Itea chinensis var. oblonga)、光叶山矾(Symplocos lancifolia)、桂北木 姜子、狗骨柴、黄线润楠(Machilus grijsii)和茜树 
表2 格氏栲天然林不同大小林窗群落系统发育多样性与物种多样性

Table 2 Phylogenetic diversity (PD) and plants taxonomic diversity of different forest gaps in Castanopsis kawakamii nature forest

\begin{tabular}{llllll}
\hline & 指数 Index & 大林窗 Large gap & 中林窗 Middle gap & 小林窗 Small gap & 非林窗 Non-gap \\
\hline 系统发育多样性 & SES.PD & $995.37 \pm 173.75^{\mathrm{a}}$ & $904.14 \pm 0.82^{\mathrm{b}}$ & $901.02 \pm 89.64^{\mathrm{b}}$ & $638.09 \pm 74.88^{\mathrm{b}}$ \\
$\begin{array}{l}\text { Phylogenetic } \\
\text { diversity }\end{array}$ & $N R I$ & $-0.231 \pm 0.213^{\mathrm{a}}$ & $-0.051 \pm 0.327^{\mathrm{a}}$ & $-0.283 \pm 0.598^{\mathrm{a}}$ & $-0.502 \pm 0.380^{\mathrm{a}}$ \\
& $N T I$ & $-0.261 \pm 0.487^{\mathrm{a}}$ & $0.577 \pm 0.546^{\mathrm{a}}$ & $1.391 \pm 0.977^{\mathrm{a}}$ & $0.473 \pm 0.769^{\mathrm{a}}$ \\
物种多样性 & Margalef & $2.72 \pm 0.44^{\mathrm{a}}$ & $2.69 \pm 0.17^{\mathrm{b}}$ & $2.24 \pm 0.42^{\mathrm{b}}$ & $1.98 \pm 0.36^{\mathrm{b}}$ \\
Species diversity & Simpson & $0.59 \pm 0.01^{\mathrm{a}}$ & $0.58 \pm 0.01^{\mathrm{a}}$ & $0.48 \pm 0.02^{\mathrm{a}}$ & $0.45 \pm 0.01^{\mathrm{a}}$ \\
& Shannon-Wiener & $1.48 \pm 0.23^{\mathrm{a}}$ & $1.59 \pm 0.05^{\mathrm{b}}$ & $1.33 \pm 0.12^{\mathrm{b}}$ & $1.27 \pm 0.08^{\mathrm{b}}$ \\
& $\begin{array}{l}\text { 物种丰富度 } \\
\text { Species richness }\end{array}$ & $11.33 \pm 2.28^{\mathrm{a}}$ & $9.01 \pm 2.24^{\mathrm{ab}}$ & $8.51 \pm 2.37^{\mathrm{b}}$ & $6.67 \pm 0.62^{\mathrm{b}}$ \\
& Pielou均匀度 & $0.43 \pm 0.08^{\mathrm{a}}$ & $0.53 \pm 0.02^{\mathrm{a}}$ & $0.46 \pm 0.01^{\mathrm{a}}$ & $0.45 \pm 0.01^{\mathrm{a}}$ \\
\hline
\end{tabular}

NRI: 净谱系亲缘关系指数; NTI: 净最近种间亲缘关系指数。同行不同字母表示不同大小林窗间系统发育多样性和物种多样性具有显著差异 $(P<0.05)$ 。

NRI, Net relatedness index; NTI, Net nearest taxa index. Different lowercases in the same row indicate significant differences of phylogenetic diversity and plants taxonomic diversity between different forest gap sizes $(P<0.05)$.

(Aidia cochinchinensis)。中林窗优势种为格氏栲、 木荷、桂北木姜子、茜树和光叶山矾。小林窗优 势种包括格氏栲、木荷、桂北木姜子、茜树和鼠 刺。非林窗优势种主要有格氏栲、狗骨柴和桂北 木姜子。大林窗优势种基本涵盖了中、小林窗和 非林窗的优势种，中、小林窗优势种种类基本一 致(附录1)。

\section{2 不同大小林窗群落物种多样性与系统发育} 多样性

由表 2 可知，林窗的 SES.PD 指数介于 901.02-995.37之间, 从大到小依次是大林窗、中 林窗和小林窗。物种多样性指数越大, SES.PD值 也越大。NRI和NTI在不同大小林窗之间无显著差 异性。群落系统发育结构在大林窗中趋于发散 $(N R I<0, N T I<0)$, 在中、小林窗和非林窗中 $N R I$ 指数与 NTI指数结果正负不一致, 无法判定该区 域群落系统发育结构发散还是聚集。

\section{3 物种多样性与系统发育多样性的相关性}

由物种多样性与系统发育多样性的相关性可 知(图1), 格氏栲林窗木本植物群落系统发育多样 性SES.PD指数与SR、Margalef和S-W指数均存在 显著正相关关系 $(P<0.05)$ 。 NRI、NTI与物种多样 性指数之间相关关系不显著。

\section{4 物种多样性与系统发育多样性的驱动因子}

去除共线性后的环境因子与群落物种多样 性、系统发育多样性和系统发育结构指数进行 GLM分析(图2，附录2)发现，林窗大小对SES.PD、
SR和Shannon-Wiener指数均存在显著正效应 $(P<$ $0.05)$ 。土壤全氮是影响系统发育结构 $(N R I 、 N T I)$

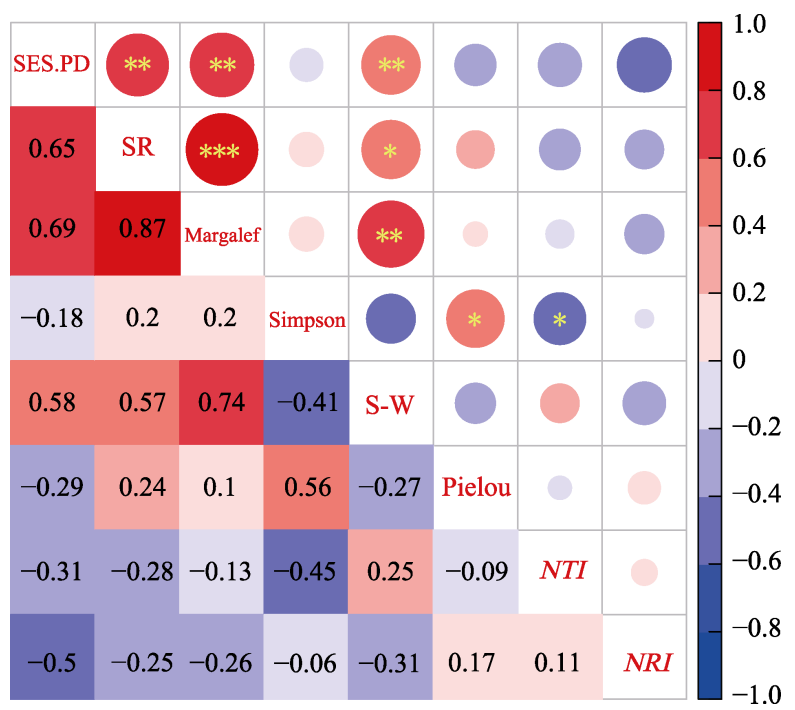

图1 格氏栲天然林群落物种多样性指数与系统发育指数 之间的相关分析。红色表示正相关, 蓝色表示负相关。颜 色越深，圆形越大，表示相关性越强。SES.PD: 标准化系 统发育多样性; SR: 物种丰富度; NRI: 净谱系亲缘关系 指数; NTI: 净最近种间亲缘关系指数; $\mathrm{S}-\mathrm{W}$ : Shannon-Wiener指数; *: $P<0.05$; **: $P<0.01$; **: $P<$ 0.001。

Fig. 1 Pearson correlation between plants taxonomic and phylogenetic indices of Castanopsis kawakamii forest communities. Red and blue indicated positive and negative correlation, respectively. The darker color and larger circle indicated a stronger correlation. SES.PD, standardization phylogenetic diversity; SR, Species richness; NRI, Net relatedness index; NTI, Net nearest taxa index; S-W, Shannon-Wiener index; *, $P<0.05$; **, $P<0.01$; ***, $P<$ 0.001 . 
(a) SES.PD

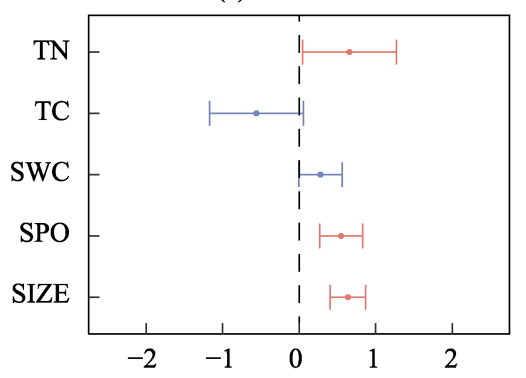

(d) SR

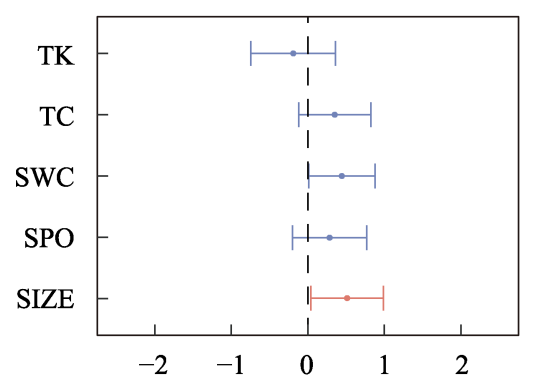

(g) Pielou (b) NTI

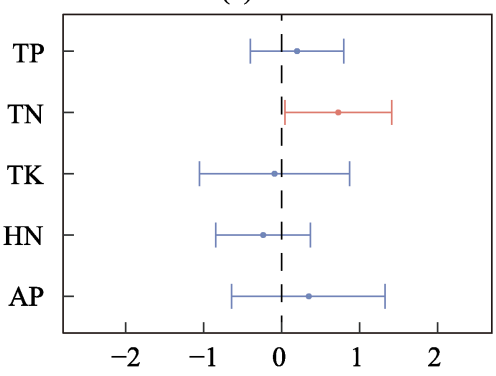

(e) Margalef

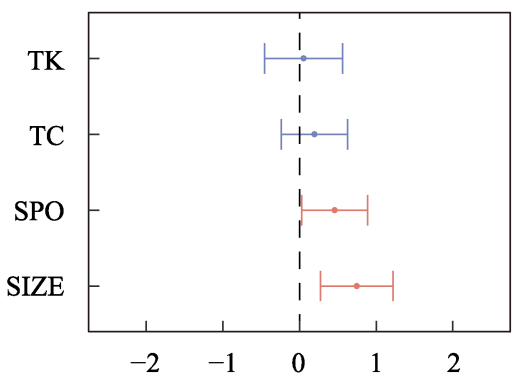

(h) S-W

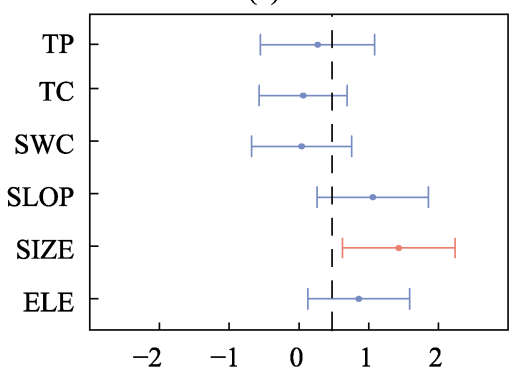

(c) NRI

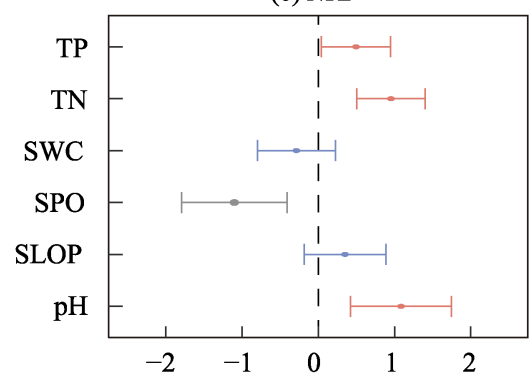

(f) Simpson

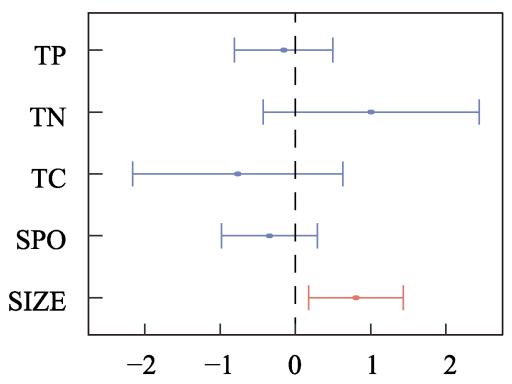

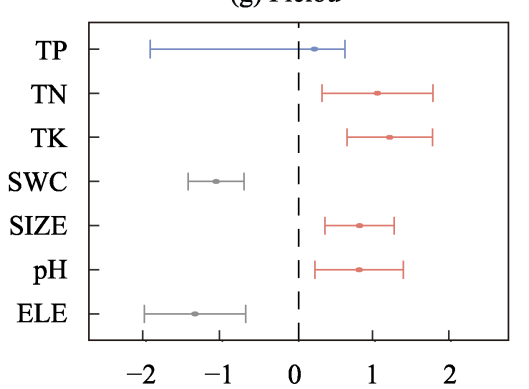

图2 环境因子对系统发育多样性、系统发育结构和物种多样性指数的影响。线条表示 $95 \%$ 置信区间, 红点表示显著正效应 $(P<0.05)$, 灰点表示显著负效应 $(P<0.05)$, 蓝点表示不显著影响。SES.PD: 标准化系统发育多样性; SR: 物种丰富度; NRI: 净谱系亲缘关系指数; NTI: 净最近种间亲缘关系指数; S-W: Shannon-Wiener指数; SIZE: 面积; ELE: 海拔; TC: 全碳; TN: 全氮; TP: 全磷; AP: 有效磷; TK: 全钾; SWC: 土壤含水量; HN: 水解氮: SPO: 坡位; SLOP: 坡度。

Fig. 2 The relative effects of environmental factors on phylogenetic diversity, phylogenetic structure and plants taxonomic index. The lines represent 95\% confidence intervals; the red dots represent significant positive effects $(P<0.05)$, and the gray dots represent significant negative effects $(P<0.05)$, the blue dots show an insignificant effect. SES.PD, Standardization phylogenetic diversity; SR, Species richness; NRI, Net relatedness index; NTI, Net nearest taxa index; S-W, Shannon-Wiener index; SIZE, Area; ELE, Elevation; TC, Total carbon; TN, Total nitrogen; TP, Total phosphorus; AP, Available phosphorus; TK, Total potassium; SWC, Soil water content; HN, Hydrolyzed nitrogen; SPO, Slope position; SLOP, Slope.

指数和SES.PD指数的重要因子, 且随土壤全氮增 加, NRI、NTI和SES.PD指数呈单调递增趋势。坡 位与SES.PD指数存在显著正效应 $(P<0.05)$, 与 $N R I$ 指数存在显著负效应 $(P<0.05)$ 。海拔和土壤含 水量与Pielou均匀度指数存在负效应 $(P<0.05)$ 。

\section{3 讨论}

\section{1 不同大小林窗的物种多样性与系统发育多} 样性

林窗面积增加或减少会影响树木幼苗生长和
群落更新(Terra et al, 2012)。格氏栲天然林物种多 样性和系统发育多样性随林窗面积增大而增加 (表2)。这是因为随着林窗面积增大，一方面增强 了光能吸收, 使地表温度上升, 促进了土壤种子 的萌发和有机质的分解(Lu et al, 2018); 另一方面, 大林窗可为物种扩散提供更多机会，提高林窗内 物种多样性(Hammond \& Pokorný，2020)。此外, 林窗边缘效应也有效促进了物种多样性增加(张 艳等，2016)。大林窗相较于中、小林窗具有更大 的边缘区域和更明显的边缘效应，其生物多样性 
也较高(刘金福等, 2003)。

由群落系统发育多样性与系统发育结构可推 断出群落构建的生态过程, 对揭示群落动态有重 要意义(Webb, 2000)。不同大小林窗群落种类组成 和物种丰富度存在差异, 大林窗物种种类与丰富 度相对较高, 其物种组成基本涵盖了中、小林窗 和非林窗, 对应的系统发育多样性指数也高于 中、小林窗。中、小林窗中物种组成基本一致, 其 对应的SES.PD指数基本相等。可见, 物种种类多 的林窗, 其对应的SES.PD指数也相对较高(表2, 附录1)。格氏栲木本植物群落系统发育结构在大 林窗中趋于发散 $(N R I<0, N T I<0)$, 表明在大林窗 中竞争作用占主导地位(Qian et al, 2020)。而中、 小林窗和非林窗中 NTI、NRI正负性不一致, 其可 能原因是中、小林窗及非林窗中, 物种组成差异 不大, 群落受生境过滤与竞争排斥综合作用, 导 致无法判定该区域群落系统发育结构是发散还是 聚集(Kress et al, 2009)。NTI与NRI指数不一致与其 对分类阶元等级的依赖以及物种在不同阶元等级 分布不均有关, 或受参数本身适用范围和敏感程 度影响, 也可能与系统发育信号强度有关(Tucker et al, 2017)。

\section{2 林窗群落的物种多样性与系统发育多样性 的相关性}

SES.PD指数与 SR、Margalef丰富度指数和 Shannon-Wiener指数呈极显著正相关 $(P<0.01)$, 与物种Simpson优势度指数和Pielou均匀度指数相 关性不显著(图1), 其可能原因是格氏栲林窗中稀 有种的物种种类多于林窗内优势种数, 且在大林 窗中更为明显; Shannon-Wiener指数对于稀有种 更加敏感(马克平等, 1995), 物种稀疏的群落中, 群落系统发育结构趋于发散, 反之则为聚集, 进 一步说明大林窗中群落系统发育结构趋于发散。 此外, 物种多样性指数如 Shannon-Wiener 、 Simpson和Pielou指数均与NTI和NRI之间无明显相 关性, 可能是由于格氏栲林窗处于发育早期, 生 态位分化较大, 资源相对较为丰富导致的(白家烨 等, 2018)。

\section{3 物种多样性与系统发育多样性的驱动因子}

土壤养分含量是影响物种多样性的关键因素 (Long et al, 2018)。随着土壤全氮含量增加, 物种
多样性会降低(Niu et al, 2014)。Lu等(2011)研究发 现氮含量与物种多样性呈显著负相关, 主要通过 改变土壤性质来影响植物生长, 进而影响到物种 多样性。林窗研究中氮含量与物种多样性呈显著 正相关, 与 $\mathrm{Lu}$ 等(2010)的研究不一致, 在于他们 主要关注郁闭的林下层植被, 但林下层植被主要 受光因素限制, 土壤氮含量可能对植物生长影响 不大, 而林窗群落光资源限制较小, 土壤氮的可 利用性对植物生长更为重要(Guo et al, 2019)。氮 存在于光合酶中, 参与植物光合作用, 除光照水 平外, 氮含量也是影响植物光合作用的重要环境 因子之一(Rho et al, 2020)。格氏栲天然林林窗土 壤全氮含量与 $N T I 、 N R I$ 和SES.PD指数呈极显著正 相关关系 $(P<0.01)$ (图2)。土壤全氮含量影响林窗 内群落系统发育结构, 这与CaO等(2020)全氮含量 对植被恢复的研究结果一致。林窗内光照强度发 生变化, 促进了植物对氮的吸收和生长, 有利于 群落物种多样性与系统发育多样性增加。

林窗形成提高了群落物种多样性, 增加物种 丰富度, 为树种更新和生存提供有利的生存空间 (Devagiri et al, 2016)。林窗面积是影响林窗群落物 种多样性和群落系统发育多样性的重要因子(图 2)。随着林窗面积增加, 昆虫和小型动物等更易被 天敌捕食, 使得林窗内种子被取食概率降低, 植 物种子存活几率升高, 进而提高林窗群落的物种 多样性与系统发育多样性, 这与李强等(2019)在 西双版纳关于林窗植物多样性研究的结果一致。

坡向、坡位是重要地形因子, 通过改变光照、 水分、温度和土壤等生态因子间接影响植物生长 发育和生物多样性(Finkel et al, 2013; 张荣等, 2020)。格氏栲天然林林窗中, 坡位是驱动SES.PD 指数重要的环境因子。林窗坡位不同, 光照以及 温度环境也存在异质性, 直接影响林窗内种子萌 发、幼苗生长和发育, 最终影响林窗内群落系统 发育多样性(Seyednasrollah \& Kumar, 2014)。

林窗海拔与Pielou均匀度指数之间存在显著 负相关关系 $(P<0.01)$ (图2)。海拔升高改变了林窗 内的温度、热量、水分等条件, 导致部分幼苗无 法适应特定环境而被淘汰, 呈现出随海拔上升均 匀度下降的趋势。该结论与宋爱云等(2006)在卧龙 自然保护区亚高山植物群落多样性的研究结果相 
反, 可能是因为本研究的格氏栲样地均处于低海 拔地区, 而卧龙自然保护区的研究样地处于中高 海拔地区。有研究表明, 海拔高度是影响群落均 匀度的重要因素: 在低海拔时, 均匀度随海拔升 高而减小，到中海拔时，则随着海拔升高而增大， 呈现出波浪上升趋势(金章利等, 2019)。

林窗形成促进了格氏栲天然林群落物种多样 性和系统发育多样性。大林窗物种多样性和系统 发育多样性显著高于中、小林窗和非林窗，且大 林窗内优势种种类基本涵盖了中、小林窗与非林 窗内优势种种类。林窗面积对物种多样性呈显著 正效应, 是驱动物种多样性变化的重要因子。土 壤全氮含量对林窗群落系统发育多样性与系统发 育结构存在显著正效应。

\section{ORCID}

何中声 (D) https://orcid.org/0000-0002-7031-7340

\section{参考文献}

Bai JY, Liu WH, Zhao BQ, Zhang Q, Guo DG (2018) Biodiversity of subalpine meadow in Heyeping of Luya Mountain, China. Journal of Applied Ecology, 29, 389-396. (in Chinese with English abstract) [白家烨, 刘 卫华, 赵冰清, 张青, 郭东罡 (2018) 芦芽山荷叶坪亚 高山草甸生物多样性. 应用生态学报, 29, 389-396.]

Bao SD (2000) Soil Agrochemical Analysis, 3rd edn. China Agriculture Press, Beijing. (in Chinese) [鲍士旦 (2000) 土壤农化分析 (第三版). 中国农业出版社, 北京.]

Buajan S, Liu JF, He ZS, Feng XP, Muhammad A (2018) Effects of gap size and locations on the regeneration of Castanopsis kawakamii in a subtropical natural forest, China. Journal of Tropical Forest Science, 30, 39-48.

Cao ZW, Fang X, Xiang WH, Lei PF, Peng CH (2020) The vertical differences in the change rates and controlling factors of soil organic carbon and total nitrogen along vegetation restoration in a subtropical area of China. Sustainability, 12, 6443.

Ci XQ, Li J (2017) Phylogenetic diversity and its application in floristics and biodiversity conservation. Biodiversity Science, 25, 175-181. (in Chinese with English abstract) [慈秀芹，李捷 (2017) 系统发育多样性在植物区系研 究与生物多样性保护中的应用。生物多样性, 25, 175-181.]

Devagiri GM, Khaple AK, Mohan S, Venkateshamurthy P, Tomar S, Arunkumar AN, Joshi G (2016) Species diversity, regeneration and dominance as influenced by canopy gaps and their characteristics in tropical evergreen forests of Western Ghats, India. Journal of Forestry Research, 27, 799-810.
Faith DP (1992) Conservation evaluation and phylogenetic diversity. Biological Conservation, 61, 1-10.

Finkel M, Fragman O, Nevo E (2013) Biodiversity and interslope divergence of vascular plants caused by sharp microclimatic differences at "Evolution Canyon II", Lower Nahal Keziv, Upper Galilee, Israel. Israel Journal of Plant Sciences, 49, 285-296.

Forest F, Grenyer R, Rouget M, Jonathan Davies T, Cowling RM, Faith DP, Balmford A, Manning JC, Procheş Ş, van der Bank M, Reeves G, Hedderson TAJ, Savolainen V (2007) Preserving the evolutionary potential of floras in biodiversity hotspots. Nature, 445, 757-760.

Gastauer M, Thiele J, Porembski S, Neri AV (2020) How do altitude and soil properties influence the taxonomic and phylogenetic structure and diversity of Brazilian páramo vegetation? Journal of Mountain Science, 17, 1045-1057.

Guo XL, Chen L, Zheng RB, Zhang K, Qiu YP, Yue HT (2019) Differences in soil nitrogen availability and transformation in relation to land use in the Napahai Wetland, Southwest China. Journal of Soil Science and Plant Nutrition, 19, 92-97.

Hammond ME, Pokorný R (2020) Preliminary assessment of effect of disturbance on natural regeneration in gaps of different sizes. Journal of Forest Science, 66, 185-196.

He ZS, Liu JF, Wu CT, Zheng SQ, Hong W, Su SJ, Wu CZ (2012) Effects of forest gaps on some microclimate variables in Castanopsis kawakamii natural forest. Journal of Mountain Science, 9, 706-714.

He ZS, Liu JF, Zheng SQ, Hong W, Wu ZY, Xu DW, Wu CZ (2012) Effects of forest gap disturbance on plant species diversity and stability in regeneration layers of Castanopsis kawakamii natural forests. Plant Science Journal, 30, 133-140. (in Chinese with English abstract) [何中声，刘金福，郑世群，洪伟，吴则焰，徐道炜，吴 承祯 (2012) 林窗对格氏栲天然林更新层物种多样性 和稳定性的影响. 植物科学学报, 30, 133-140.]

Huang JX, Zheng FY, Mi XC (2010) Influence of environmental factors on phylogenetic structure at multiple spatial scales in an evergreen broad-leaved forest of China. Chinese Journal of Plant Ecology, 34, 309315. (in Chinese with English abstract) [黄建雄, 郑凤英, 米 湘成 (2010) 不同尺度上环境因子对常绿阔叶林群落 的谱系结构的影响. 植物生态学报, 34, 309-315.]

Hubbell SP, Foster RB, O’Brien ST, Harms KE, Condit R, Wechsler B, Wright SJ, de Lao SL (1999) Light-gap disturbances, recruitment limitation, and tree diversity in a Neotropical forest. Science, 283, 554-557.

Hu LY, Li JS, Wu XP, Yan BQ, Zhu JJ, Luo JW, Xiao NW (2010) Reviews on methods of measuring geometric characteristics of forest gaps involving gap size, gap shape, and the height of canopy trees surrounding the gap. Acta Ecologica Sinica, 30, 19141919. (in Chinese with English abstract) [胡理乐, 李俊生, 吴晓莆, 间伯前, 朱 
教君, 罗建武, 肖能文 (2010) 林窗几何特征的测定方 法. 生态学报, 30, 1911-1919.]

Jin ZL, Liu GP, Zhou MT, Xu WN (2019) Elevation characteristics of grassland community diversity and effect of soil physical and chemical properties in karst mountain grassland. Ecology and Environmental Sciences, 28, 661-668. (in Chinese with English abstract) [金章利, 刘高鹏, 周明涛, 许文年 (2019) 喀斯特山地草地群落 多样性海拔特征及土壤理化性质特征. 生态环境学报, 28, 661-668.]

Kress WJ, Erickson DL, Jones FA, Swenson NG, Perez R, Sanjur O, Bermingham E (2009) Plant DNA barcodes and a community phylogeny of a tropical forest dynamics plot in Panama. Proceedings of the National Academy of Sciences, USA, 106, 18621-18626.

Li Q, Wang B, Deng Y, Lin LX, Dawa ZX, Zhang ZM (2019) Correlation between spatial distribution of forest canopy gaps and plant diversity indices in Xishuangbanna tropical forests. Biodiversity Science, 27, 2ZZ5. (in Chinese with English abstract) [李强, 王彬, 邓云, 林露 湘, 达低扎喜, 张志明 (2019) 西双版纳热带雨林林窗 空间分布格局及其特征指数与林窗下植物多样性的相 关性. 生物多样性, 27, 273-285.]

Liu JF, Hong W, Li JQ, Lin RF (2003) Gap edge effect of Castanopsis kawakamii community. Chinese Journal of Applied Ecology, 14, 142426. (in Chinese with English abstract) [刘金福, 洪伟, 李俊清, 林荣福 (2003) 格氏栲群落林窗边缘效应研究. 应用生态学报, 14, 1421-1426.]

Lohbeck M, Poorter L, Martínez-Ramos M, Rodriguez-Velázquez J, van Breugel M, Bongers F (2014) Changing drivers of species dominance during tropical forest succession. Functional Ecology, 28, 1052-1058.

Long C, Yang XB, Long WX, Li DH, Zhou W, Zhang H (2018) Soil nutrients influence plant community assembly in two tropical coastal secondary forests. Tropical Conservation Science, 11, 194008291881795.

Lu DL, Wang GG, Yu LZ, Zhang T, Zhu JJ (2018) Seedling survival within forest gaps: The effects of gap size, within-gap position and forest type on species of contrasting shade-tolerance in Northeast China. Forestry Research, 91, 470-479.

Lu XK, Mo JM, Gilliam FS, Yu GR, Zhang W, Fang YT, Huang J (2011) Effects of experimental nitrogen additions on plant diversity in tropical forests of contrasting disturbance regimes in southern China. Environmental Pollution, 159, 2228-2235.

Ma KP, Huang JH, Yu SL, Chen LZ (1995) Plant community diversity in Dongling Mountain, Beijing, China: II. Species richness, evenness and species diversities. Acta Ecologica Sinica, 15, 268277. (in Chinese) [马克平, 黄 建辉, 于顺利, 陈灵芝 (1995) 北京东灵山地区植物群 落多样性的研究. II. 丰富度、均匀度和物种多样性. 生
态学报, 15, 268-277.]

Mao XG, Zhu L, Fan WY (2020) Object-oriented automatic identification of forest gaps using digital orthophoto maps and LiDAR data. Canadian Journal of Remote Sensing, 46, 177-192.

Mori AS, Fujii S, Kitagawa R, Koide D (2015) Null model approaches to evaluating the relative role of different assembly processes in shaping ecological communities. Oecologia, 178, 261-273.

Myers JA, Chase JM, Jiménez I, Jørgensen PM, Araujo-Murakami A, Paniagua-Zambrana N, Seidel R (2013) Beta-diversity in temperate and tropical forests reflects dissimilar mechanisms of community assembly. Ecology Letters, 16, 151-157.

Niu KC, Choler P, de Bello F, Mirotchnick N, Du GZ, Sun SC (2014) Fertilization decreases species diversity but increases functional diversity: A three-year experiment in a Tibetan alpine meadow. Agriculture, Ecosystems and Environment, 182, 106-112.

Pio DV, Broennimann O, Barraclough TG, Reeves G, Rebelo AG, Thuiller W, Guisan A, Salamin N (2011) Spatial predictions of phylogenetic diversity in conservation decision making. Conservation Biology, 25, 1229-1239.

Qian H, Deng T, Jin Y, Mao LF, Zhao D, Richlefs RE (2019) Phylogenetic dispersion and diversity in regional assemblages of seed plants in China. Proceedings of the National Academy of Sciences, USA, 116, 23192-23201.

Qian H, Hao ZQ, Zhang J (2014) Phylogenetic structure and phylogenetic diversity of angiosperm assemblages in forests along an elevational gradient in Changbaishan, China. Journal of Plant Ecology, 7, 154-165.

Qian H, Jin Y (2014) An updated megaphylogeny of plants, a tool for generating plant phylogenies and an analysis of phylogenetic community structure. Journal of Plant Ecology, 9, 233-239.

Qian H, Zhang J, Sandel B, Jin Y (2020) Phylogenetic structure of angiosperm trees in local forest communities along latitudinal and elevational gradients in eastern North America. Ecography, 43, 419-430.

R core team (2019) R: A Language and Environment for Statistical Computing. R Foundation for Statistical Computing. Vienna.

Rho H, Doty SL, Kim SH (2020) Endophytes alleviate the elevated $\mathrm{CO}_{2}$-dependent decrease in photosynthesis in rice, particularly under nitrogen limitation. Journal of Experimental Botany, 71, 707-718.

Seyednasrollah B, Kumar M (2014) Net radiation in a snow-covered discontinuous forest gap for a range of gap sizes and topographic configurations. Journal of Geophysical Research, 119, 10323-10342.

Song AY, Liu SR, Shi ZM, Dong LS, Liu JT (2006) Study on species diversity of subalpine meadow communities in Wolong Nature Reserve. Forest Research, 19, 767-772. 
(in Chinese with English abstract) [宋爱云, 刘世荣，史 作民，董林水，刘京涛 (2006) 卧龙自然保护区亚高山 草甸植物群落物种多样性研究. 林业科学研究, 19, 767-772.]

Sun Q, Lu JB, Zhang FF, Xu GF (2009) Plant species diversity in relation to island size. Acta Ecologica Sinica, 29, 2195-2202. (in Chinese with English abstract) [孙雀, 卢剑波, 张凤凤, 徐高福 (2009) 植物物种多样性与岛 屿面积的关系. 生态学报, 29, 2195-2202.]

Tucker CM, Cadotte MW, Carvalho SB, Davies TJ, Ferrier S, Fritz SA, Grenyer R, Helmus MR, Jin LS, Mooers AO, Pavoine S, Purschke O, Redding DW, Rosauer DF, Winter M, Mazel F (2017) A guide to phylogenetic metrics for conservation, community ecology and macroecology. Biological Reviews, 92, 698-715.

Terra NAR, Araújo GM, Giroldo AB, Silva PPF (2013) Gap area and tree community regeneration in a tropical semideciduous forest. In: Tropical Forests (ed. Sudarshana P), pp. 139-154. IntechOpen, London.

Wang JX, Zhang YP (2002) A review on within-gap micro-environmental heterogeneity and species' response. Journal of Nanjing Forestry University (Natural Sciences Edition), 26(1), 69-74. (in Chinese with English abstract) [王进欣, 张一平 (2002) 林窗微环境异质性及物种的 响应. 南京林业大学学报(自然科学版), 26(1), 69-74.]

Webb CO (2000) Exploring the phylogenetic structure of ecological communities: An example for rain forest trees. The American Naturalist, 156, 145-155.

Webb CO, Ackerly DD, McPeek MA, Donoghue MJ (2002) Phylogenies and community ecology. Annual Review of
Ecology and Systematics, 33, 475-505.

Yao JY, Wu XB, Sun QH, Wu X, Yao XL, Hao JF, Qi JQ (2018) Effects of canopy gap size on understory species diversity and biomass in a Pinus massoniana plantation in western Sichuan. Chinese Journal of Applied and Environmental Biology, 24, 214-220. (in Chinese with English abstract) [姚俊宇, 伍炫蓓, 孙千惠, 吴霞, 姚小 兰, 郝建锋, 齐锦秋 (2018) 林窗大小对川西马尾松人 工林林下物种多样性和生物量的影响. 应用与环境生 物学报, 24, 214-220.]

Zhang R, Yu FY, Zhou RH, Dong HJ, Wang M, Ye X, Hao JF (2020) Effects of slope position and aspect on structure and species diversity of shrub community in the Jiajin Mountains, Sichuan Province, China. Chinese Journal of Applied Ecology, 31, 2507-2514. (in Chinese with English abstract) [张荣, 余飞燕, 周润惠, 董洪君, 王 敏, 叶金縏, 建锋 (2020) 坡向和坡位对四川夹金山灌 从群落结构与物种多样性特征的影响. 应用生态学报, 31, 2507-2514.]

Zhang Y, Zhang DJ, Li X, Liu H, Zhang MJ, Yang WQ, Zhang J (2016) Edge effects of forest gap in Pinus massoniana plantations on the decomposition of leaf litter recalcitrant components of Cinnamomum camphora and Toona ciliata. Chinese Journal of Applied Ecology, 27, 1116-1124. (in Chinese with English abstract) [张艳, 张 丹桔, 李勋, 刘华, 张明锦, 杨万勤, 张健 (2016) 马尾 松人工林林窗边缘效应对樟和红椿调落叶难降解物质 分解的影响. 应用生态学报, 27, 1116-1124.]

(责任编委: 蔵润国 责任编辑: 黄祥忠)

\section{附录 Supplementary Material}

附录1 不同大小林窗的群落物种组成

Appendix 1 Species composition of plant community in various areas of forest gaps http://www.biodiversity-science.net/fileup/PDF/2020399-1.pdf

附录2 利用广义线性模型分析物种多样性、系统发育多样性指数与环境因子的关系

Appendix 2 Using generalized linear model to analyze the relationship between plants taxonomic, phylogenetic diversity index and environmental factors

http://www.biodiversity-science.net/fileup/PDF/2020399-2.pdf 
陈博, 江蓝, 谢子扬, 李阳娣, 李佳葍, 李梦佳, 魏晨思, 邢聪, 刘金福, 何中声 (2021) 格氏栲天然林林窗植物物种多样性与系统发育多样性. 生物多样性, 29 (4): 439-448. http://www.biodiversity-science.net/CN/10.17520/biods.2020399

\section{附录1 不同大小林窗的群落物种组成}

Appendix 1 Species composition of plant community in various areas of forest gaps

\begin{tabular}{|c|c|c|c|}
\hline 林窗/非林窗 & 种名 Species & 多度 Abundance & 耐阴特性 Characteristics of shade tolerance \\
\hline \multirow{19}{*}{ 大林窗 } & 格氏栲 Castanopsis kawakamii & 102 & 中性 \\
\hline & 桂北木姜子 Litsea subcoriacea & 85 & 耐阴 \\
\hline & 鼠刺 Itea chinensis var. oblonga & 72 & 耐阴 \\
\hline & 木荷 Schima superba & 46 & 喜光 \\
\hline & 黄线润楠 Machilus grijsii & 32 & 喜光 \\
\hline & 茜树 Aidia cochinchinensis & 21 & 喜光 \\
\hline & 狗骨柴 Diplospora dubia & 19 & 耐阴 \\
\hline & 光叶山矾 Symplocos lancifolia & 16 & 喜光 \\
\hline & 山矾 Symplocos sumuntia & 14 & 喜光 \\
\hline & 小叶蚊母树 Distylium buxifolium & 13 & 中性 \\
\hline & 虎皮楠 Daphniphyllum oldhami & 11 & 耐阴 \\
\hline & 新木姜子 Neolitsea aurata & 11 & 喜光 \\
\hline & 细枝柃 Eurya loquaiana & 9 & 耐阴 \\
\hline & 华南木姜子 Litsea greenmaniana & 7 & 喜光 \\
\hline & 毛冬青 Ilex pubescens & 7 & 喜光 \\
\hline & 马尾松 Pinus massoniana & 6 & 喜光 \\
\hline & 山杜英 Elaeocarpus sylvestris & 5 & 耐阴 \\
\hline & 红皮䊅果茶 Camellia octopetala & 5 & 耐阴 \\
\hline & 冬青 Ilex chinensis & 3 & 耐阴 \\
\hline \multirow{19}{*}{ 中林窗 } & 桂北木姜子 Litsea subcoriacea & 71 & 耐阴 \\
\hline & 格氏栲 Castanopsis kawakamii & 38 & 中性 \\
\hline & 木荷 Schima superba & 46 & 喜光 \\
\hline & 光叶山矾 Symplocos lancifolia & 20 & 喜光 \\
\hline & 细枝柃 Eurya loquaiana & 14 & 耐阴 \\
\hline & $\begin{array}{l}\text { 香港新木姜子 Neolitsea cambodiana var. } \\
\text { glabra }\end{array}$ & 13 & 耐阴 \\
\hline & 赤楠 Syzygium buxifolium & 11 & 耐阴 \\
\hline & 黄线润楠 Machilus grijsii & 11 & 喜光 \\
\hline & 狗骨柴 Diplospora dubia & 6 & 耐阴 \\
\hline & 山杜英 Elaeocarpus sylvestris & 6 & 耐阴 \\
\hline & 马尾松 Pinus massoniana & 5 & 喜光 \\
\hline & 日本杜英 Elaeocarpus japonicus & 3 & 耐阴 \\
\hline & 红皮踈果茶 Camellia octopetala & 3 & 耐阴 \\
\hline & 新木姜子 Neolitsea aurata & 3 & 喜光 \\
\hline & 米槠 Castanopsis carlesii & 3 & 中性 \\
\hline & 毛冬青 Ilex chinensis & 3 & 喜光 \\
\hline & 树参 Dendropanax dentigerus & 2 & 耐荫 \\
\hline & 山黄皮 Randia cochinchinensis & 2 & 耐阴 \\
\hline & 闽楠 Phoebe bournei & 3 & 喜光 \\
\hline \multirow{4}{*}{ 小林窗 } & 桂北木姜子 Litsea subcoriacea & 74 & 耐阴 \\
\hline & 茜树 Aidia cochinchinensis & 46 & 喜光 \\
\hline & 木荷 Schima superba & 43 & 喜光 \\
\hline & 格氏栲 Castanopsis kawakamii & 38 & 中性 \\
\hline
\end{tabular}


陈博, 江蓝, 谢子扬, 李阳娣, 李佳葍, 李梦佳, 魏晨思, 邢聪, 刘金福, 何中声 (2021) 格氏栲天然林林窗植物物种多样性与系统发育多样性. 生物多样性, 29 (4): 439-448. http://www.biodiversity-science.net/CN/10.17520/biods.2020399

\begin{tabular}{|c|c|c|c|}
\hline 林窗/非林窗 & 种名 Species & 多度 Abundance & 耐阴特性 Characteristics of shade tolerance \\
\hline \multirow{33}{*}{ 非林窗 } & 鼠刺 Itea chinensis var. oblonga & 35 & 耐阴 \\
\hline & 榕叶冬青 Ilex ficoidea & 14 & 耐阴 \\
\hline & 黄线润楠 Machilus grijsii & 13 & 喜光 \\
\hline & 山矾 Symplocos sumuntia & 12 & 喜光 \\
\hline & 光叶山矾 Symplocos lancifolia & 11 & 喜光 \\
\hline & 毛冬青 Ilex chinensis & 11 & 喜光 \\
\hline & 狗骨柴 Diplospora dubia & 11 & 耐阴 \\
\hline & 木姜叶柯 Lithocarpus litseifolius & 8 & 中性 \\
\hline & 新木姜子 Neolitsea aurata & 5 & 喜光 \\
\hline & 日本杜英 Elaeocarpus japonicus & 4 & 耐阴 \\
\hline & 细枝柃 Eurya loquaiana & 3 & 耐阴 \\
\hline & 白花苦灯笼 Tarenna mollissima & 3 & 中性 \\
\hline & $\begin{array}{l}\text { 香港新木姜子 Neolitsea cambodiana var. } \\
\text { glabra }\end{array}$ & 2 & 耐阴 \\
\hline & 小叶赤楠 Syzygium grijsii & 2 & 耐阴 \\
\hline & 马尾松 Pinus massoniana & 1 & 喜光 \\
\hline & 狗骨柴 Diplospora dubia & 33 & 耐阴 \\
\hline & 桂北木姜子 Litsea subcoriacea & 23 & 耐阴 \\
\hline & 格氏栲 Castanopsis kawakamii & 21 & 中性 \\
\hline & 油茶 Camellia oleifera & 14 & 喜光 \\
\hline & 黄线润楠 Machilus grijsii & 13 & 喜光 \\
\hline & 树参 Dendropanax dentigerus & 12 & 耐阴 \\
\hline & 木荷 Schima superba & 11 & 喜光 \\
\hline & 小叶石楠 Photinia parvifolia & 10 & 耐阴 \\
\hline & 新木姜子 Neolitsea aurata & 8 & 喜光 \\
\hline & 光叶山矾 Symplocos lancifolia & 7 & 喜光 \\
\hline & 山矾 Symplocos sumuntia & 7 & 喜光 \\
\hline & 白花苦灯笼 Tarenna mollissima & 6 & 中性 \\
\hline & 毛冬青 Ilex chinensis & 5 & 耐阴 \\
\hline & 尖叶水丝梨 Distyliopsis dunnii & 4 & 耐阴 \\
\hline & 香叶树 Lindera communis & 4 & 喜光 \\
\hline & 小叶蚊母树 Distylium buxifolium & 3 & 耐阴 \\
\hline & $\begin{array}{l}\text { 香港新木姜子 Neolitsea cambodiana var. } \\
\text { glabra }\end{array}$ & 2 & 耐阴 \\
\hline & 细枝柃 Eurya loquaiana & 2 & 耐阴 \\
\hline
\end{tabular}

物种按照各区域内物种丰富度进行排序。

Species are sorted according to the species richness in each area. 